\title{
Food Ordering Restaurant Android Application
}

\author{
K V Aishwarya ${ }^{1}$, Aishwarya Chavan ${ }^{2}$, Sedjwick Drego ${ }^{3}$, Akshay Chavan ${ }^{4}$, Prof .P.P Bastawade ${ }^{5}$ \\ All India Shri Shivaji Memorial Society's Polytechnic Pune, India ${ }^{1,2,3,4,5}$
}

\begin{abstract}
Nowadays popularity of restaurants is ever increasing. Accordingly, the number of restaurants is growing. The purpose of proposed system is to develop an Restaurant Ordering System which enables customer to place their order at any time and any place. The reason to develop the system is due to the issues faced by the restaurants such as peak hour-long queue issues. Therefore, this system enhances the speed and standardization of taking the order from the customers. Furthermore, it also extends and delivers customer's satisfaction especially to the hectic customer or reaching the customer who face transport problems to go to the restaurants. The customer is able to customize their order without errors in communication between the customer and the person taking the order. He can also choose a manual addressing option which means that he/her can place an order which will be delivered right to his/her home even if they are outside at the time of placing the order.
\end{abstract}

Keywords: Notification, XAMPP, Android studio.

\section{INTRODUCTON}

Nowadays restaurants are one of the favorite premises popularity of restaurants has increased. The proposed system is an android based restaurant application. The new user will have to sign up providing his details which will be stored in the database, and an already existing user can login by providing us his user id and password. The user can choose what he wants to have from the menu. The customer will have an entire menu to choose from. He can increase or decrease the number of items he wants to have and add them to the cart. Then the customer can checkout and that's it his order will be delivered to his doorstep.

The main advantage is that the customers don't have to wait in long queues to order what they want to have and then wait for some more time to get the food.

Also there will be no misunderstandings whatsoever as there no interaction is taking place between the customer and the person taking the order because it will be completely computerized.

To create this project we have used android studio v2.2.3. This android studio has a brilliant environment which makes it easy for programming.

\section{Android studio-}

It is the official integrated development environment (IDE) for the Android platform. It was announced on May 16, 2013 at the Google I/O conference. Android Studio is freely available under the Apache License 2.0. Android Studio was in early access preview stage starting from version 0.1 in May 2013, then entered beta stage starting from version 0.8 which was released in June 2014. The first stable build was released in December 2014, starting from version 1.0. Based on JetBrains' IntelliJ IDEA software, Android Studio is designed specifically for Android development. It is available for download on
Windows, macOS and Linux, and replaced Eclipse Android Development Tools (ADT) as Google's primary IDE for native Android application development.

\section{Features}

New features are expected to be rolled out with each release of Android Studio.

The following features are provided in the current stable version.

- Gradle-based build support

- Android-specific refactoring and quick fixes

- Lint tools to catch performance, usability, version compatibility and other problems

- ProGuard integration and app-signing capabilities

- Template-based wizards to create common Android designs and components

- A rich layout editor that allows users to drag-and-drop UI components, option to preview layouts on multiple screen configurations.

- Support for building Android Wear apps

- Built-in support for Google Cloud Platform, enabling integration with Google Cloud Messaging and App Engine.

- An Android Virtual Device that is used to run and debug apps.

\section{EXISTING SYSTEM}

There are other types of restaurant applications. But they support information about hundreds and thousands of restaurants. Online restaurants are very limited and most of them use only websites for online ordering of food. We have created and application which the user just have to download on his/her android smartphone and in a few minutes he can easily order food of his choice. 


\section{PROPOSED SYSTEM}

The proposed system is different from the existing systems. Yes, it is a restaurant application, but unlike other applications of this kind, our application is only supported for one restaurant. All the user has to do is download the application on his/her android mobile phone, log in (if he is an existing user) or sign up (if he is a new user) By entering the details required, select the items from the given menu, checkout, provide his address while checking out and that's it. Food that he ordered will be delivered within no time and with no mistakes whatsoever.

\section{BASIC IDEA}

This application is designed, keeping in mind the customer's comfort. Now a days, because of the hectic life that people lead, they do not have time to go to a restaurant, order food, pay for it, then wait for some more time to finally get their food.

This application is for the customers' comfort, where he can order food from anywhere and it will be delivered right to the door step of the address that he has given. As you can see in the above figure, the user will see a welcome page. If he is a new user, he will have to sign up.
At that time he will have to enter his details and choose a user id and a password. The password should consist of more than 6 characters and the user cannot leave any fields empty. He will also be asked to provide his name. If the user is an existing user then he will simply have to login by entering his user id and password. This user id and password will be matched with the one existing in the database which was entered by the same user at the time of sign up. Then the menu page will be displayed and the user will have to select at least one item. The user cannot proceed further without selecting at least one item from the menu.

When he places his order, an alert box is displayed for the confirmation of the menu. After that the total amount will be displayed and the user will be asked to enter the address to which he wants the food to be delivered. The address is mandatory and the user cannot proceed further without entering the address. For user's safety purpose, the address is not saved in the database and the user will be asked to enter his address each time he makes an order. After that the Thank you page will be displayed and the user can either sign out or he can go back to the menu page and start a whole new order. Whichever option he chooses, the details from the previous menu will be erased for safety purposes.

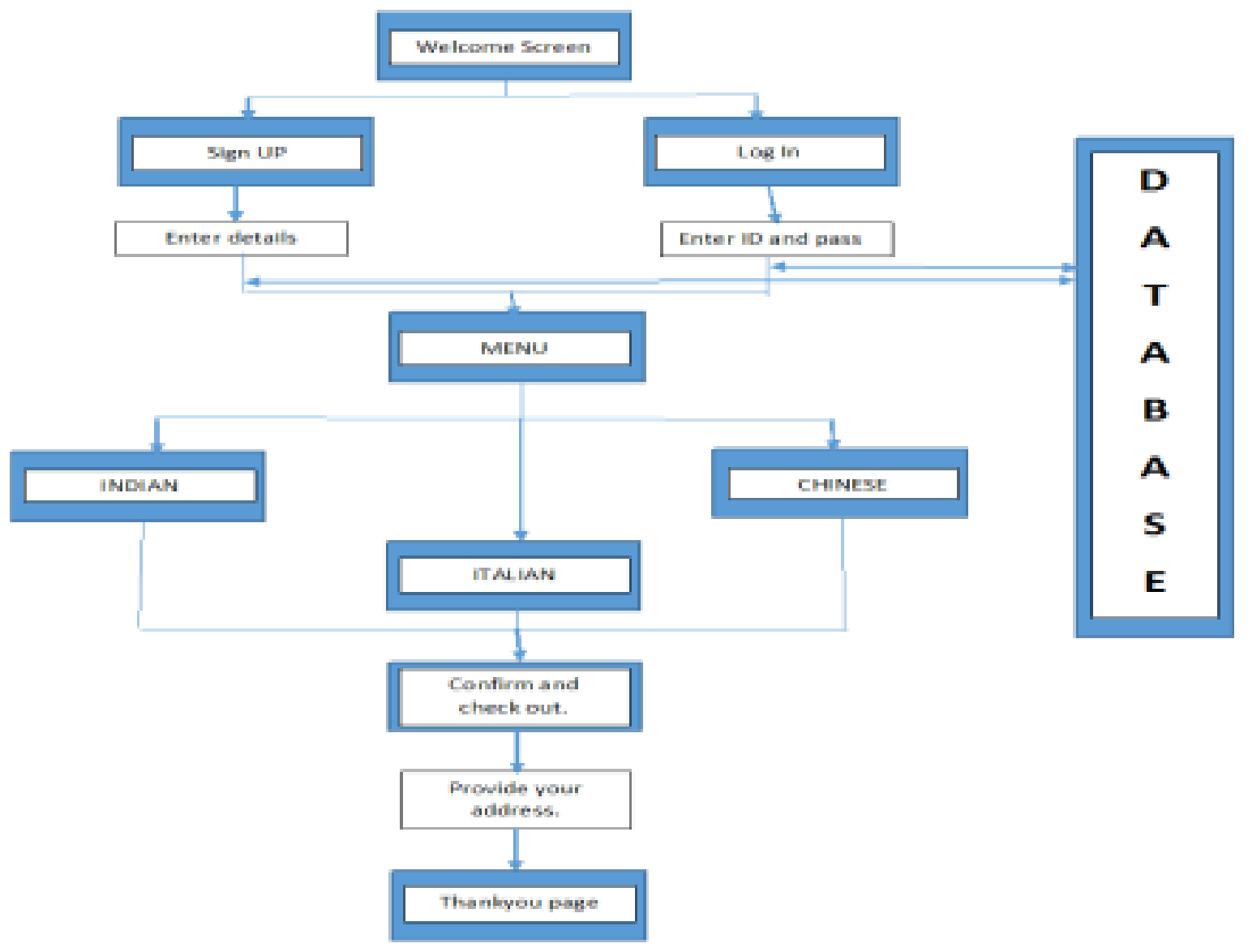

Fig 1: Architecture of the system. 


\section{IMPLEMENTATION}

\section{A. Android Application}

This is the first page of our application. This screen is a splash screen which will be displayed for 4 seconds.

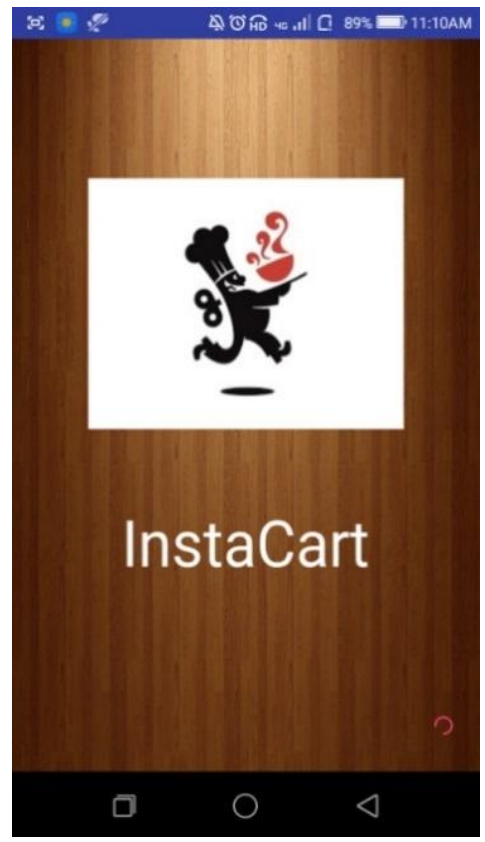

Fig 2. Welcome Page

This is the screen where the new user will have to select the sign up button and the existing user will have to select the log in button.

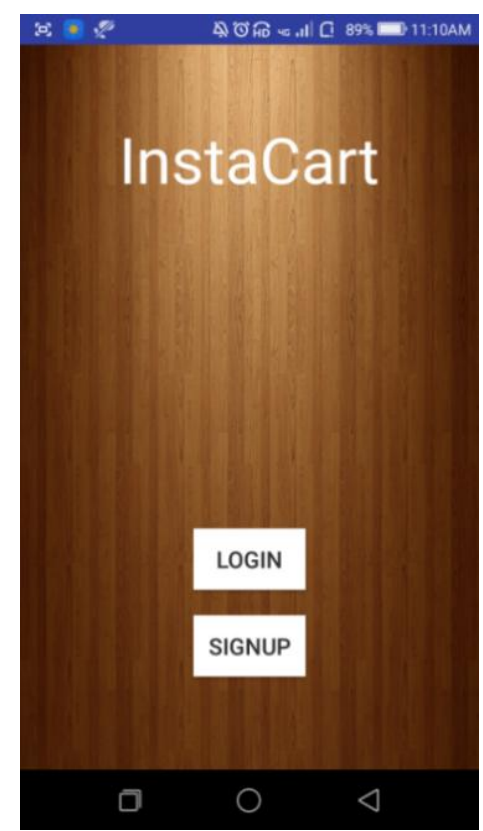

Fig 3. Login / Signup

This is the signup page where the new user will have to enter his details in order for him to go ahead and use the application.

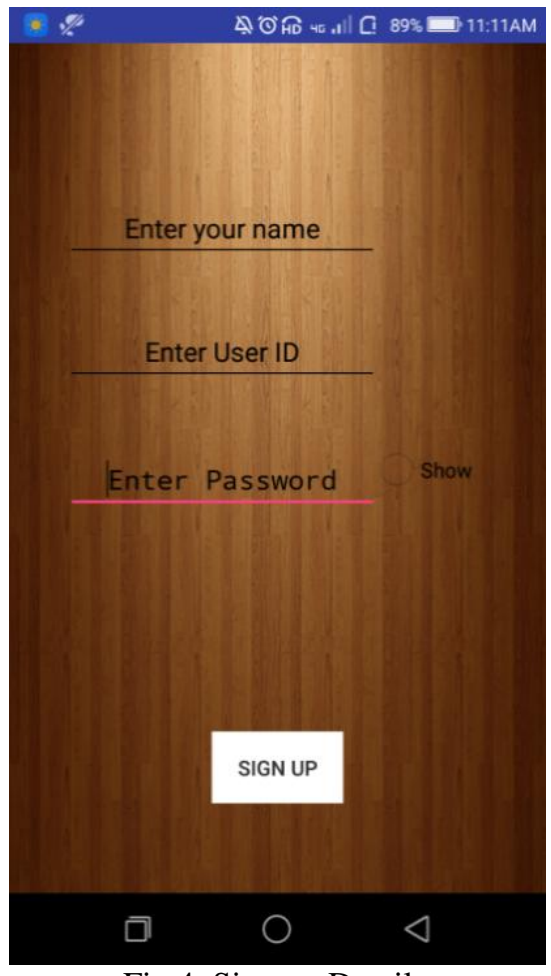

Fig 4. Sign up Details

If you are an existing user, you need to $\log$ in.

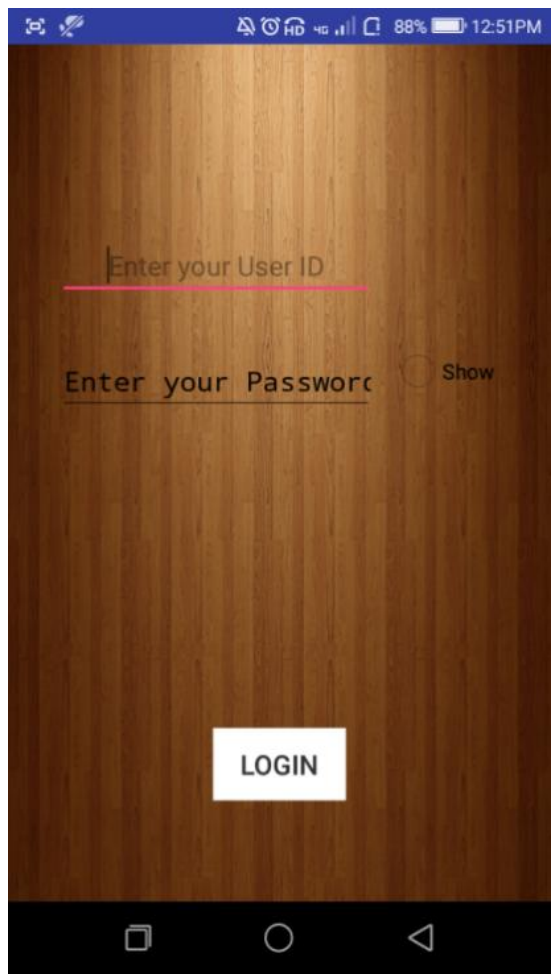

Fig 5. Log in Details

This is the MENU page which is displayed after the welcome page, it consists of different dishes that the customer can choose from and there are plus $(+)$ and minus (-) to increment or decrement the number of items. 


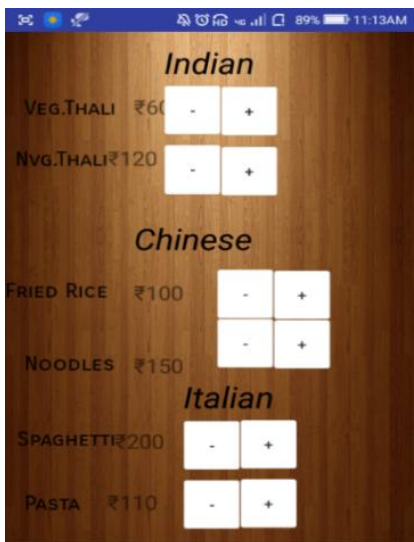

PROCEED TO PAYMENT

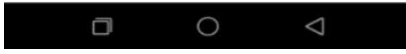

Fig 6. Menu

Total amount is displayed. User needs to enter his address.

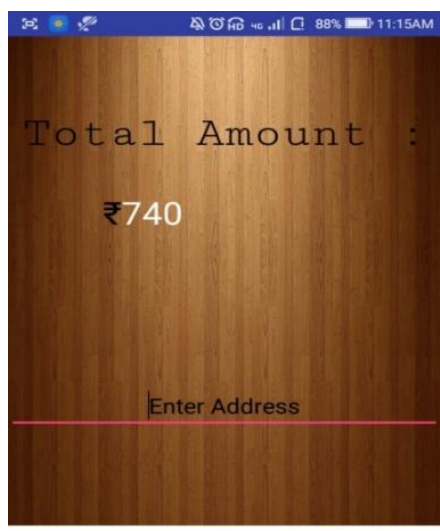

CHECKOUT

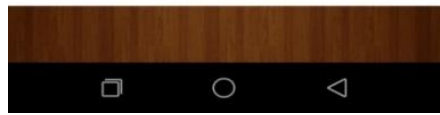

Fig 8. Address

Confirmation of order is mandatory.

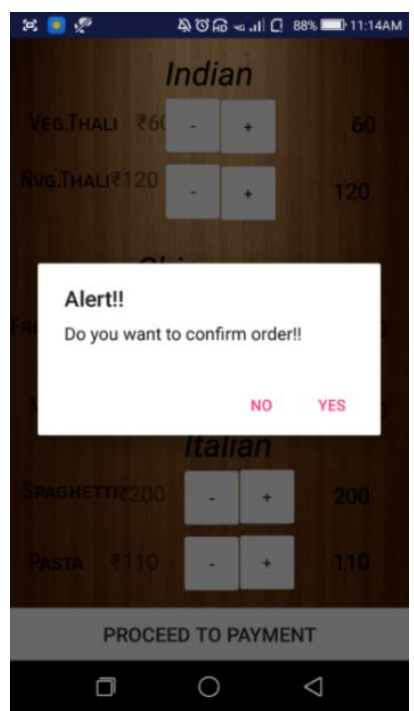

Fig 7. Confirmation
Thank you message is displayed, you can either sign out or go back to the menu.

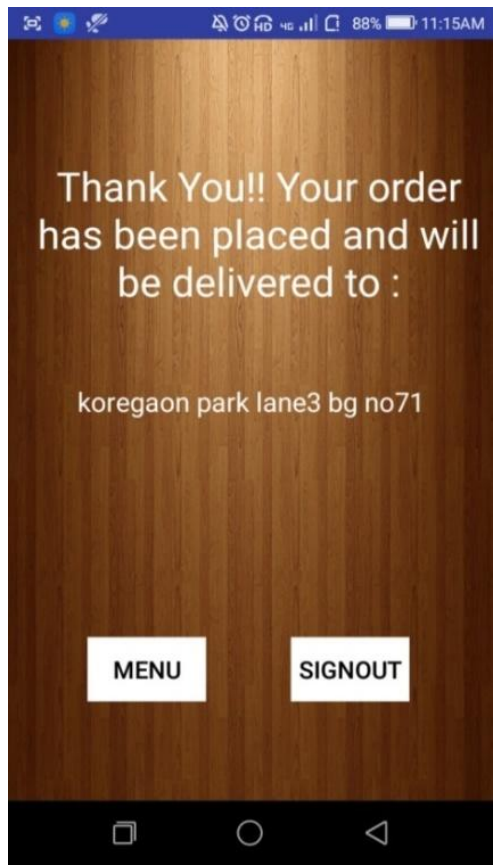

Fig 9.Thankyou page

\section{FEATURES OF PROPOSED SYSTEM}

- No interaction between the customer and the restaurant has to be made.

- The whole ordering process is online.

- The customer can give whichever address he feels comfortable with.

- Less time is consumed.

- People don't have to wait in queues especially during the peak hours.

\section{CONCLUSION}

This application is designed by keeping in mind the customer's comfort. Now a days, because of the hectic life that people lead, they do not have time to go to a restaurant, order food, pay for it, then wait for some more time to finally get their food. This application is for the customers' comfort, where he can order food from anywhere and it will be delivered right to the door step of the address that he has given. We conclude by saying that this is going to be a very useful application once it's put into use, and will save a lot of people's time.

\section{FUTURE WORKS}

This application can be used by a restaurant. Then they can display their own menu and take control. The application will then be uploaded to google play and the customers can easily download the application and use it. Once the application is uploaded to Google Play, people can download this application. Then thy simply have to 
sign in and choose from the Menu displayed, provide their Address and that's it. The restaurant will directly home deliver their food to the address mentioned. In future, maybe we can also add a payment option of card. Some people find Cash on delivery hard as they rarely keep any cash at home. Card payment option will provide these kind of customers to do online payment and not worry about having cash at home for Cash on delivery option.

\section{ACKNOWLEDGEMENT}

This work is completed under the guidance of Prof. Prajakta Bastawade we express our gratitude towards them for their continuous support on this research. We would also like to thank the reviewers for their suggestions to improve this paper.

\section{REFERENCES}

[1] Patel Krishna M., Patel Palak P. , Raj Nirali R., Patel Lalit A. -"Automated Food Ordering System"

[2] Younghoon Chang, Siew Fan Wong, Priscilla a/p Sugumar and MuthukumaranMaruthappa "-Determinants of Consumer Intention to Continue Using TableTop Tablet Ordering Systems in Restaurant Businesses."

[3] Julian Meichsner - "Evaluation of Interactive Tablets for Improving Customer Service in Restaurants"

[4] Mir RoomiRahil, Rajesh Mahind, SaurabhChavan, TanumayDhar Feedback

[5] Prof V.B. Dhore, SurabhiThakar, PrajaktaKulkarni, RasikaThorat "Digital Table Booking and Food Ordering System Using Android Application."

[6] AshutoshBhargave, NiranjanJadhav, Apurva Joshi, PrachiOke, Prof. Mr. S. R Lahane -“ Digital Ordering System for Restaurant Using Android."

[7] VarshaChavan, PriyaJadhav, SnehalKorade and PriyankaTeli "Implementing Customizable Online Food Ordering System Using Web Based Application"

[8] ReshamShinde, PriyankaThakare, NehaDhomne, SushmitaSarkar "Integration of Technology in Restaurants using Android".

[9] ShwetaShashikantTanpure, Priyanka R. Shidankar, Madhura M. Joshi - "Automated Food Ordering System with Real-Time Customer Feedback"

[10] Prof V.B. Dhore, SurabhiThakar, PrajaktaKulkarni, RasikaThorat "Digital Table Booking and Food Ordering System Using Android Application". 